\title{
Correction to: Fluvastatin sensitizes pancreatic cancer cells toward radiation therapy and suppresses radiation- and/or TGF- $\beta$-induced tumor-associated fibrosis
}

Debasish Mohapatra, Biswajit Das (D), Voddu Suresh (D), Deepti Parida, Aliva Prity Minz, Usharani Nayak, Amlan Priyadarshee Mohapatra, Rajeeb K. Swain and Shantibhusan Senapati (i)

(c) The Author(s), under exclusive licence to United States and Canadian Academy of Pathology 2021

Laboratory Investigation (2022) 102:215-216; https://doi.org/10.1038/s41374-021-00707-1

Correction to: Laboratory Investigation https://doi.org/10.1038/ s41374-021-00690-7, published online 12 November 2021

The original version of this article unfortunately contained a mistake. Just after publication of this article the authors noticed an error in the Fig 2B. The representative immunofluorescence phospho- $\mathrm{\gamma H} 2 \mathrm{AX}$ image of BxPC3 [24 h; Flu $(5 \mu \mathrm{M})+2 \mathrm{~Gy}$; Fig 2B] inadvertently has got replaced with the actual image of UN-KC6141 [ 6 h; Flu $(5 \mu \mathrm{M})+2$ Gy; Fig 2 A]. The corrected figure is shown below and the corrected version doesn't affect the results and conclusion of the article. All the authors sincerely apologize for this mistake and agree with the author's correction. 
Fig. 2

A)

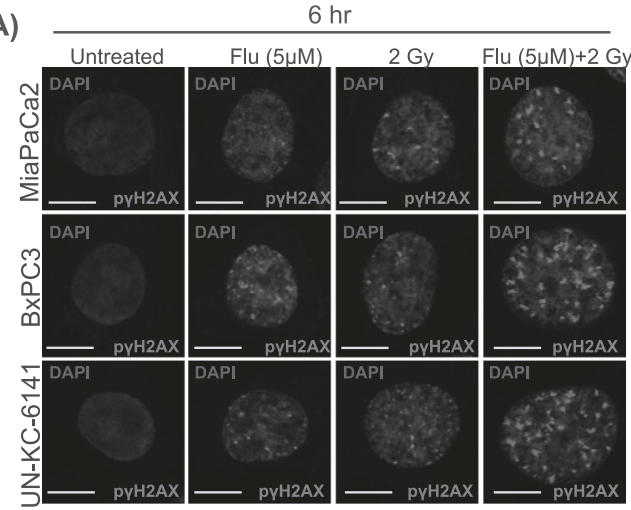

MiaPaCa2 (6 hr)

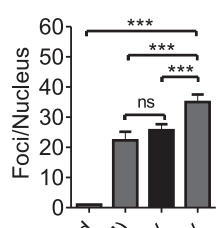

एक जा क्ष
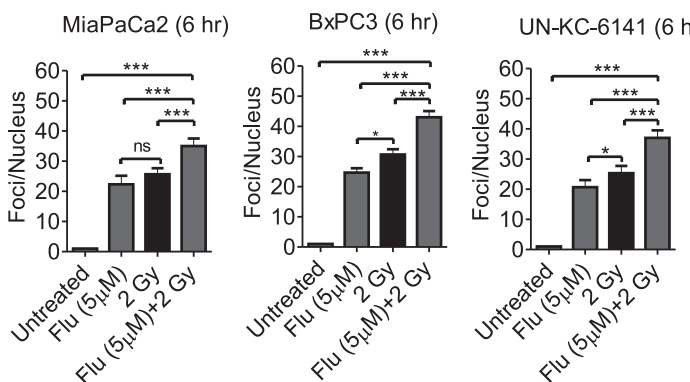

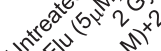

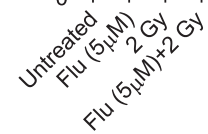

C)
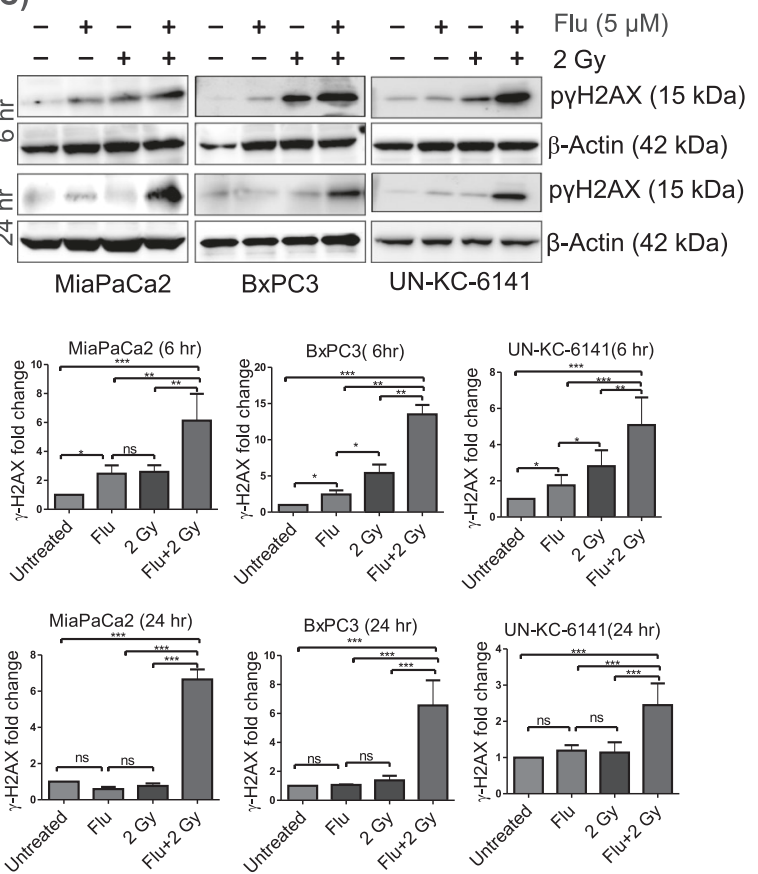

D)
B)
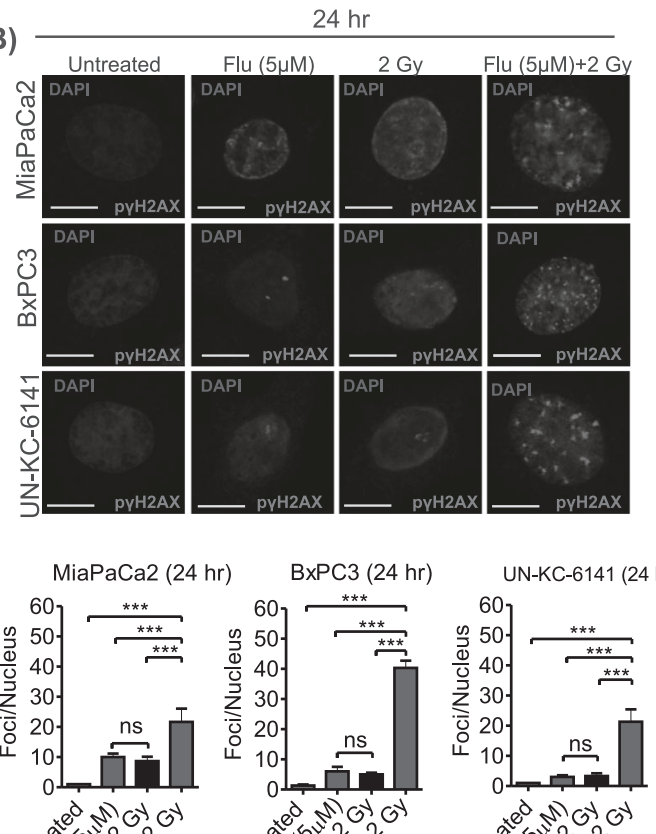
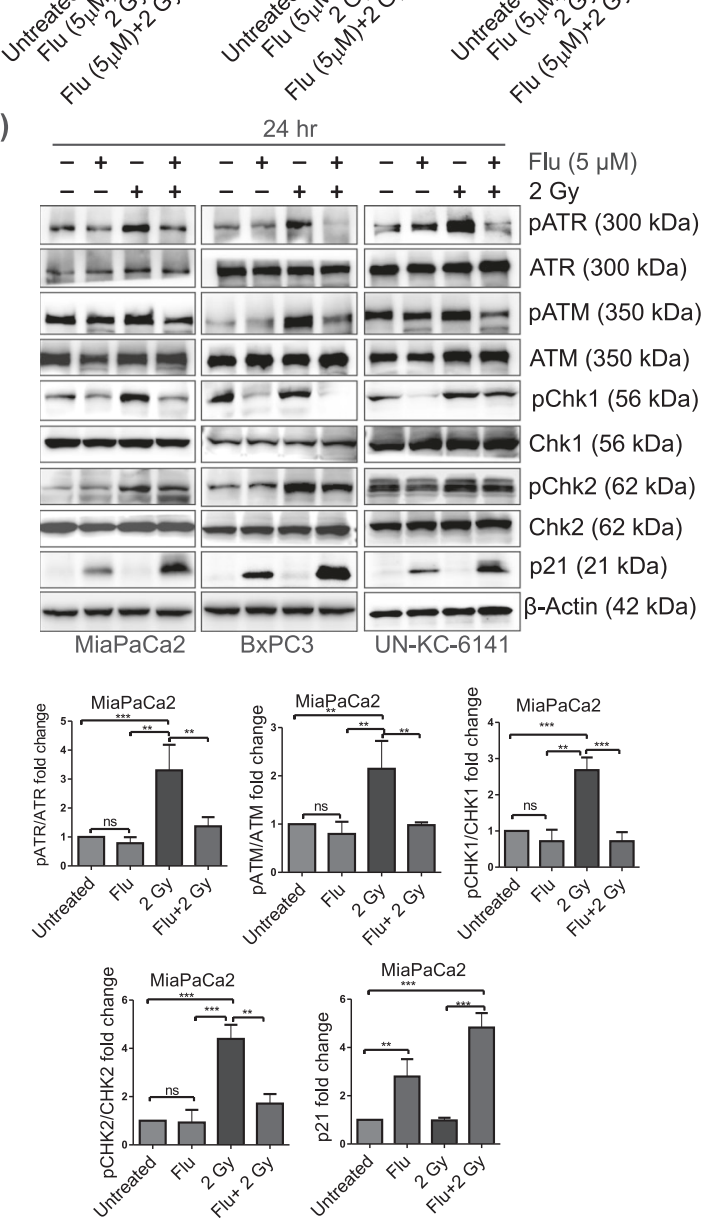